\title{
CLINICAL RESEARCH ARTICLE Dilated hypertrophy: a distinct pattern of cardiac remodeling in preterm infants
}

\author{
Nilkant S. Phad ${ }^{1,2}$, Koert de Waal ${ }^{1,2}$, Carl Holder $^{3}$ and Christopher Oldmeadow ${ }^{3}$
}

BACKGROUND: Young adults born preterm have remodeled hearts, i.e., altered cardiac shape and size with impaired cardiac function. At present, the natural history and pattern of prematurity related cardiac remodeling are not clearly established. The aim of this study was to compare the left ventricle (LV) geometry and function of preterm infants at 36 weeks postmenstrual age (PMA) with gestation matched newborn infants.

METHODS: LV end diastolic volume index (LV EDVI), LV mass index (LVMI), relative wall thickness (RWT), and sphericity index (SI) were prospectively obtained with echocardiography. LV geometry was classified according to the Gaasch method. LV function was assessed by determining ejection fraction (EF), longitudinal strain (LS), mitral annulus systolic motion (s'), and estimated LV filling pressure (E/e').

RESULTS: Eighty-three preterm infants between 23 and 29 weeks gestation, and 40 infants of 36 weeks gestation at birth were analysed. LV EDVI, LVMI, SI, LS, s', and E/e' were higher in preterm group while RWT and EF were comparable between groups. LV showed normal geometry in $55.4 \%$, physiological enlargement in $23 \%$ and dilated hypertrophy in $21.6 \%$ preterm infants.

CONCLUSION: At 36 week, preterm infants have significantly dilated, hypertrophied, and more spherical LV with impaired diastolic function compared with PMA matched newborn infants.

Pediatric Research (2020) 87:146-152; https://doi.org/10.1038/s41390-019-0568-4

\section{INTRODUCTION}

Exposure of immature organ systems to suboptimal extrauterine environment following preterm birth, can lead to altered organ development and increased risk of long term morbidity and mortality. ${ }^{1-4}$ Recent evidence suggests that similar to brain, lungs, and eyes, cardiac development is also adversely affected by preterm birth. ${ }^{3-8}$ Reduced number of cardiac muscle cells due to an early switch of the fetal hyperplastic pattern of cardiomyocyte growth to the postnatal hypertrophic pattern, combined with a prolonged exposure to an altered hemodynamic load following preterm birth can lead to cardiac remodeling, defined as changes in myocardial architecture, cardiac size, shape, and function. ${ }^{9-12}$

Several human and animal studies show that preterm birth is associated with cardiac remodeling. $8,11,13$ Young adults, born preterm, have significantly shorter and thicker left ventricle (LV) with impaired cardiac function compared with young adults born at term. ${ }^{8,14}$ Children, born extremely preterm, are reported to have significantly smaller LV with altered function compared with children born at term. ${ }^{15}$ In a large population based study from Sweden, Carr et al. noted a 17 times higher incidence of heart failure in children and young adults born extremely preterm. ${ }^{6}$ Although cardiac remodeling after preterm birth is widely reported, its natural history is not well established. Clear understanding of its origin, pattern and progression over time could prove useful in identifying infants at risk of adverse long term cardiovascular outcomes. Currently, there is not enough data to suggest or refute presence cardiac remodeling in preterm infants during the neonatal intensive care period. ${ }^{16}$
The aim of this study was to explore if the LV of preterm infants showed evidence of remodeling at 36 weeks postmenstrual age (PMA). Based on the current understanding that preterm infants face both increased preload and afterload compared with a fetus, we hypothesized that their LV will be dilated and hypertrophied. At present, it is also not known whether the preterm heart has a typical geometric pattern. In adults, certain patterns of cardiac remodeling are independent predictors of adverse cardiovascular outcomes. ${ }^{1-21}$ Our secondary aim was to classify LV geometry of preterm infants into the currently established patterns of cardiac remodeling. ${ }^{18-20}$

\section{METHODS}

This prospective observational study was conducted in the neonatal unit of the John Hunter Children's Hospital from January 2015 to June 2018. Ethics approval for this study was obtained from the local Human Research Ethics Committee.

\section{Participants}

The preterm group consisted of infants $<30$ weeks gestation at birth who were admitted to the neonatal intensive care unit during the study period. The control group included infants born at 36 weeks gestation, who were well and stayed with their mothers on the postnatal ward. Infants with congenital anomalies were excluded from both groups. Infants with the following antenatal complication were excluded from the control group: intrauterine growth restriction (IUGR), maternal diabetes mellitus, pregnancy with hypertensive disorders, premature rupture of

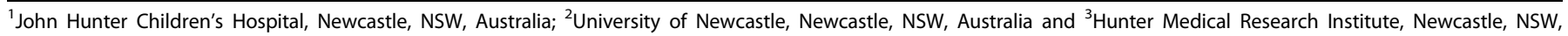
Australia

Correspondence: Nilkant S. Phad (nilkant.phad2@health.nsw.gov.au)

Received: 25 February 2019 Revised: 30 July 2019 Accepted: 25 August 2019

Published online: 7 September 2019 


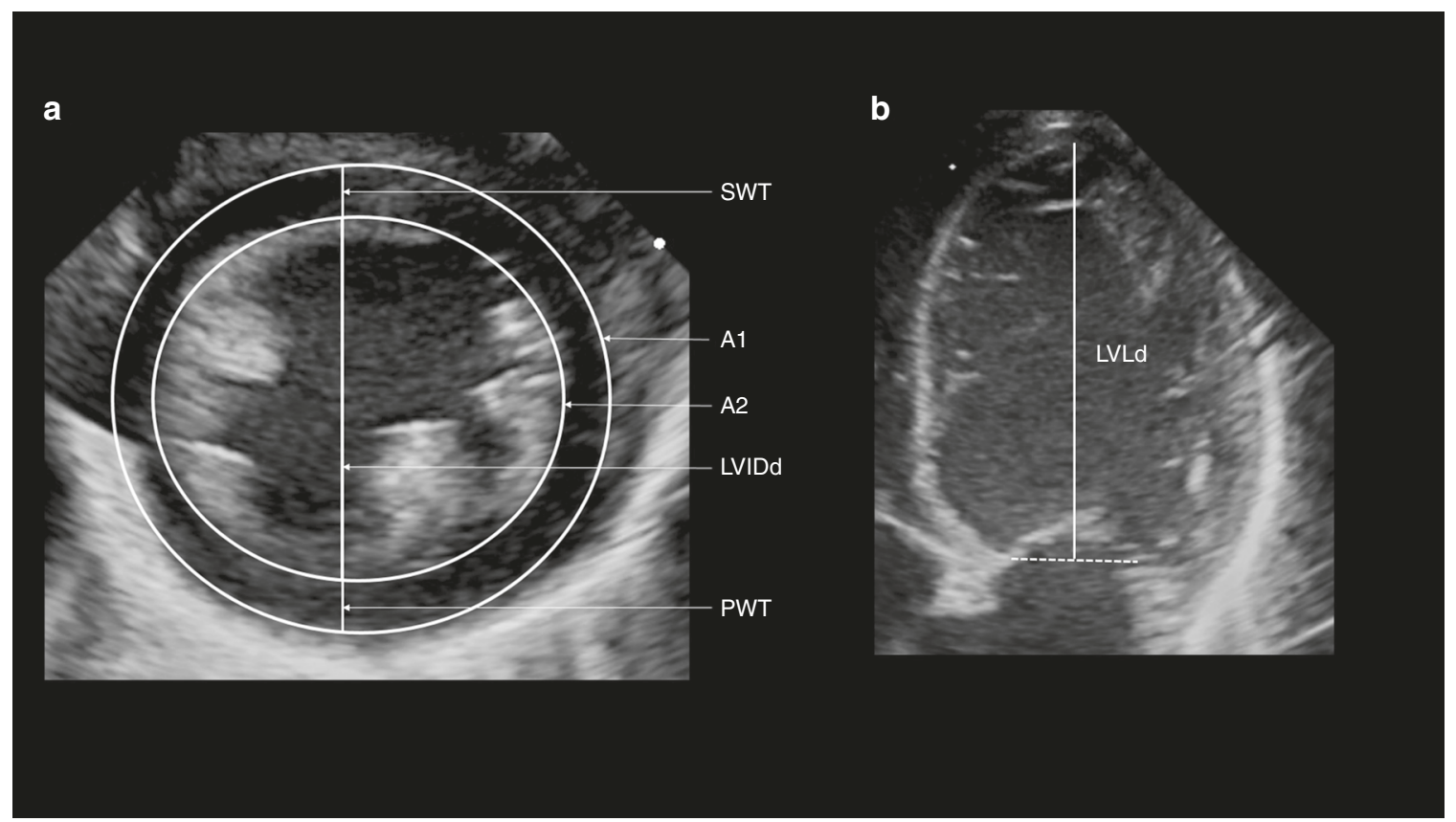

Fig. 1 Determination of LV Mass using area-length method. a Measurement of LV myocardial area from total LV area (A1) and LV cavity area (A2) in a parasternal short axis image at the level of papillary muscles. SWT septal wall thickness, PWT posterior wall thickness, LVIDd LV internal diameter at end diastole. b Measurement of LV length at end diastole (LVL) in an apical 4 chamber image

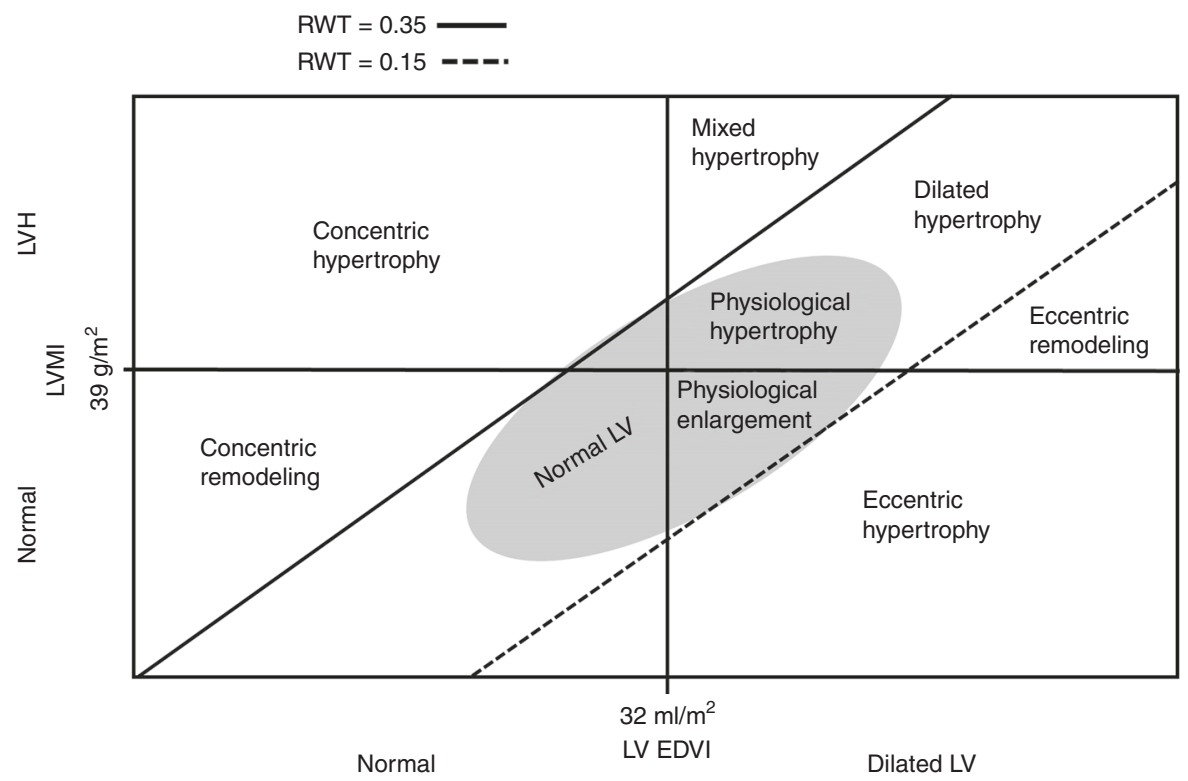

Fig. 2 LV geometric patterns classified according to LV mass index (LVMI), LV end diastolic volume index (LV EDVI), and relative wall thickness (RWT). [Adapted from Marwick et al. with permission] ${ }^{17}$

membranes for more than 7 days, oligohydramnios, polyhydramnios, histological chorioamnionitis, abnormal umbilical Doppler ultrasound study, monochorionic twining, perinatal fetal blood loss, perinatal hypoxic ischemia, and maternal substance abuse.

After an informed consent, cardiac ultrasound scans were performed at 36 weeks PMA in the preterm infants, and on postnatal day 3 in the control group subjects. Demographic details and data about the clinical condition of the infants at the time of scan were collected from their observation charts.

Image acquisition

A $12-\mathrm{MHz}$ phased-array transducer was used with an EPIQ $5 \mathrm{G}$ echocardiographic scanner (Philips Medical Systems, the
Netherlands). Images were acquired from five cardiac cycles triggered by the $\mathrm{R}$ wave and stored at acquired frame rate around $120 \mathrm{~Hz}$. Standard transthoracic echocardiographic views were obtained, and measurements were performed according to the recommendations of the American Society of Echocardiography. ${ }^{22}$ Images were stored on a server for offline analysis at a dedicated work station using Tomtec Image Arena version 4.6 and Cardiac Performance Analysis version 1.3.0.91 (Tomtec, Unterschliessheim, Germany).

Image analysis

LV mass (LVM) was determined using the area-length method from the parasternal short axis view at the level of papillary muscle 
and the apical 4 chamber view. Total area (A1) and cavity area (A2) of the LV were obtained by tracing the epicardial and endocardial border respectively at end diastole in the short axis view with the papillary muscles excluded from the endocardial tracing (Fig. 1a). The thickness of the myocardium $(T)$ was then calculated from the difference between the LV epicardial and endocardial radius. LV length (LVL) was measured from the midpoint of the line joining the mitral annulus hinge points to the apical endocardium of the $\mathrm{LV}$ at end diastole in the 4 chamber view (Fig. 1b). LVM was calculated using the formula $L V M=1.05\{[5 / 6 \times A 1(L V L+T)]-$ $[5 / 6 \times A 2(L V L)]\}$, where the number 1.05 represents the density of LV myocardial muscle. ${ }^{22}$

$L V$ relative wall thickness (RWT) and LVM to volume (M/V) ratio were used to determine the LV concentricity, an indicator of relative proportions of LV cavity size and myocardial wall. RWT was calculated from LV PWT and internal diameter (LVIDd) obtained from the parasternal short axis view at end diastole (Fig. 1a) using the formula RWT $=(2 \mathrm{X}$ PWT $) /$ LVIDd. LV end diastolic volume (EDV) was determined from the apical 4 chamber view using the method of disks and the LV M/V ratio was calculated using LVM and EDV. LV sphericity index (SI) was determined from the ratio of LVIDd and LVL. LVM and LV EDV were indexed to body surface area for comparison between the groups.

We used the geometry grid proposed by Marwick et al. to describe and classify patterns of LV geometry (Fig. 2). ${ }^{20}$ The geometry grid is a diagrammatic illustration of the Gaasch classification which provides a stepwise approach to classify LV geometry and cardiac remodeling and helps in understanding adaptation of the LV to both pressure and volume overload situations. ${ }^{18}$ These patterns of cardiac remodeling have been prospectively tested in population based studies to independently prognosticate the risk of heart failure and mortality in adults. ${ }^{17,21}$ Although not validated for outcomes in preterm infants, this established classification should be applicable to preterm infants as it acknowledges the impact of a variety of altered hemodynamic loading conditions on cardiac remodeling and utilizes commonly used parameters of LV geometry. ${ }^{5,7,15}$ LV geometry is first classified as dilated or not dilated by using LV end diastolic volume indexed to body surface area (LV EDVI). The LV is further classified as hypertrophied or not hypertrophied by using the LVM indexed to body surface area (LVMI). The RWT is subsequently used to establish whether the relative proportions of the LV PWT and LV cavity diameter are preserved despite changes in LV size. The preterm LV was labeled as dilated if the LV EDVI was more than 2 standard deviations above the mean of the control group and as hypertrophied if the LVMI was more than 2 standard deviations above the mean of the control group. A RWT within 2 standard deviations of mean RWT of the control group was considered normal.

LV function was assessed by using conventional and myocardial deformation parameters. LV systolic function was assessed from apical 4 chamber and 3 chamber images using ejection fraction (EF) by the method of disks, 2D speckle tracking-derived peak longitudinal strain (LS), and tissue Doppler-derived mitral septal annulus systolic velocity $\left(s^{\prime}\right) .^{22-24}$ LV diastolic function was assessed by estimating LV filling pressure using the Ee' ratio, obtained by dividing the trans mitral peak early diastolic Doppler blood flow velocity $(E)$ by the septal mitral annular peak early diastolic tissue Doppler velocity $\left(\mathrm{e}^{\prime}\right)$.

Statistical analysis

We calculated that 40 infants per group would give $80 \%$ power to detect a consistent difference of $20 \%$ in the left ventricular mass between groups at the $5 \%$ level of significance assuming $10 \%$ prevalence of LV hypertrophy in the preterm group. The normality of the data was tested using mean, median, and interquartile range and an independent $t$-test was used to compare the groups.
To ascertain the factors that contributed to cardiac remodeling, we used univariate and multivariate analysis. Regression modeling was used for the continuous outcomes and binomial models were used for the dichotomous outcomes. Backward stepwise regression was used to select the variables in the model. All the covariates of interest were added to the model and removed individually, starting with the covariates with highest $p$-value. Remaining coefficients were checked as the covariates were removed to insure that none of them differed by more than 20\%, which would have been an indication of confounding. To evaluate the model fit as the covariates were removed, the Akaike information criterion (AIC) and Bayesian information criterion (BIC) were used to assess the model as variables were removed individually. Both the AIC and BIC give an indication as to whether the model has improved its fit (in comparison with the previous model) with the removal of a singular variable. The covariates under examination were infant's PMA, sex, birth weight (BW), IUGR, exposure to a patent ductus arteriosus (PDA) $>1.5 \mathrm{~mm}$ for more than 14 days, systolic blood pressure, diastolic blood pressure, antenatal complications (maternal diabetes and or hypertension), postnatal steroids administration, bronchopulmonary dysplasia (BPD), and a complex postnatal clinical course (prolonged mechanical ventilation $>2$ days and/or BPD and/or postnatal steroids). There were three cardiac remodeling outcomes of interest: LV dilatation (LV EDVI $>32 \mathrm{ml} / \mathrm{m}^{2}$ ), LV hypertrophy (LVMI $>39 \mathrm{~g} /$ $\mathrm{m}^{2}$ ), and LV dilated hypertrophy (LV EDVI > $32 \mathrm{ml} / \mathrm{m}^{2}+\mathrm{LVMI}>$ $39 \mathrm{~g} / \mathrm{m}^{2}$ ).

Operator reliability of LV EDV, LVM, and RWT was tested using the interclass correlation coefficient (ICC) and Bland-Altman analysis to determine the bias and 95\% limits of agreement of the measurements. To assess intraobserver and interobserver reliability, measurements were repeated in 25 randomly chosen infants by the primary observer (two sets of measures) and by a second blinded observer (one set of measures). All analyses were conducted using SAS Version 9.4 (SAS Institute Inc., Cary, NC, USA). A $p$-value $<0.05$ was considered statistically significant.

\section{RESULTS}

The study included 83 (44 male) preterm infants and 40 (19 male) control infants. The baseline characteristics of the infants are presented in Table 1. Maternal hypertension, maternal diabetes mellitus, abnormal umbilical Doppler blood flows, preterm rupture of membranes $>7$ days, histological chorioamnionitis, and IUGR were observed in 10 (12\%), 8 (10\%), 11 (13\%), 9 (11\%), 6 (7\%), and

Table 1. Baseline characteristics of the participants

\begin{tabular}{|c|c|c|}
\hline & $\begin{array}{l}\text { Control group } \\
(N=40)\end{array}$ & $\begin{array}{l}\text { Very preterm group } \\
(N=83)\end{array}$ \\
\hline Gestation at birth (weeks) & $36(0.3)$ & $27(1.5)^{*}$ \\
\hline Weight at birth (g) & $2570(340)$ & $980(255)^{*}$ \\
\hline Gestation at scan (weeks) & $36.4(0.1)$ & $36.1(0.1)$ \\
\hline Weight at scan $(\mathrm{kg})$ & $2.59(0.34)$ & $2.48(0.39)$ \\
\hline $\begin{array}{l}\text { Body surface area at scan } \\
\left(\mathrm{m}^{2}\right)\end{array}$ & $0.190(0.014)$ & $0.180(0.016)$ \\
\hline Heart rate (beats/min) & $134(15)$ & $156(17)^{*}$ \\
\hline $\begin{array}{l}\text { Systolic blood } \\
\text { pressure }(\mathrm{mmHg})\end{array}$ & $68(9)$ & $71(8)$ \\
\hline $\begin{array}{l}\text { Diastolic blood } \\
\text { pressure }(\mathrm{mmHg})\end{array}$ & $42(8)$ & $41(9)$ \\
\hline
\end{tabular}


Table 2. Comparison of the parameters of left ventricle geometry and function

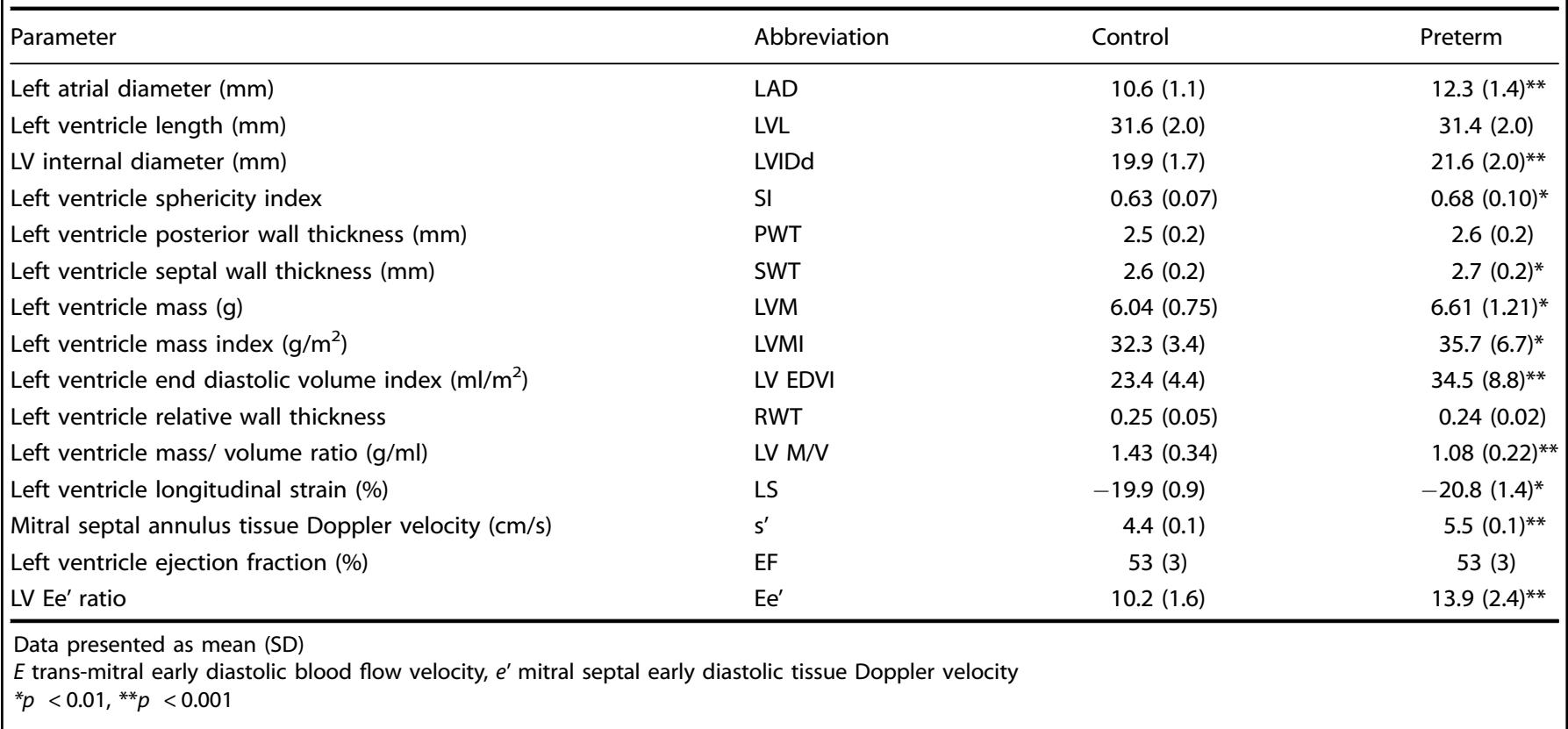

Table 3. Intra and interobserver reliability of LV mass (LVM), end diastolic volume (LV EDV), and relative wall thickness (RWT)

\begin{tabular}{lllllll}
\hline & \multicolumn{2}{l}{ Intraobserver } & & & \multicolumn{2}{l}{ Interobserver } \\
\cline { 2 - 5 } & ICC & bias & $95 \%$ LOA & & ICC & bias \\
\hline LVM (g) & 0.95 & 0.4 & -0.5 to 0.2 & 0.90 & 0.7 & -2.9 to 1.4 \\
LV EDV (ml) & 0.98 & 0.02 & -0.15 to 0.2 & 0.95 & -0.01 & -0.65 to 0.63 \\
LV RWT & 0.88 & -0.001 & -0.005 to 0.005 & 0.83 & -0.004 & -0.06 to 0.05 \\
\hline
\end{tabular}

Bias estimated from repeat and observer effects and interclass correlation (ICC) from variance components

LVM left ventricle mass, LV EDV left ventricle end diastolic volume, LV RWT left ventricle relative wall thickness, $95 \%$ LOA $95 \%$ limits of agreement

$9(11 \%)$ infants in the preterm group, respectively. During their neonatal intensive care stay, 25 (30\%) preterm infants were exposed to a PDA of $>1.5 \mathrm{~mm}$ for more than 14 days, 13 (15.6\%) received postnatal dexamethasone, and 40 (48\%) developed BPD. A PDA at 36 weeks PMA was found in two infants in preterm group and none in the control group.

Table 2 shows the parameters of LV geometry and LV function in the preterm and control groups. The preterm LV was wider with a comparable length leading to a more spherical shape compared with the control infants.

The intra and interoperator reliability was excellent for LV EDV and LVM, and good for RWT (Table 3).

The LV was dilated in $44.6 \%$ and hypertrophied in $21.6 \%$ of the preterm infants. The increase in the LV EDVI was more pronounced than the increase in the LVMI as indicated by significantly lower LV mass/volume ratio in the preterm group (Fig. 3). The RWT of the preterm group was comparable with the control group.

Using the LV geometry grid, $55.4 \%$ preterm infants were noted to have normal LV geometry and $23 \%$ showed LV enlargement with normal LVMI and RWT (physiological enlargement). LV remodeling was seen in $21.6 \%$ preterm infants and dilated hypertrophy was the only pattern of remodeling (Fig. 4). Preterm infants showed impairment of LV diastolic function with a significantly higher estimated LV filling pressure. The EF was comparable between the two groups with a small but statistically significant difference in LS and s'.

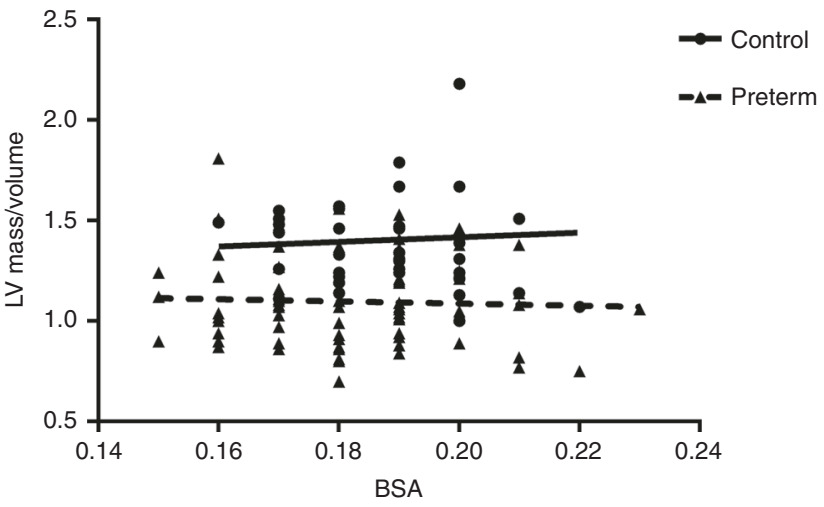

Fig. 3 LV mass/volume ratio in relation to body surface area (BSA) in control and preterm infants at 36 weeks postmenstrual age

In univariate analysis, cardiac remodeling did not show a significant association with any of the tested variables (Table 4). In the multivariate regression analysis, isolated LV dilatation was significantly associated with female sex (OR 3.1; 95\% Cl 1.0-9.6, p: 0.04 ) and a complex postnatal clinical course (OR 6.4; $95 \% \mathrm{Cl}$ 1.6-25, p: 0.007), while IUGR was associated with dilated hypertrophy (OR 9.5; 95\% Cl 0.84-107, p: 0.06). 


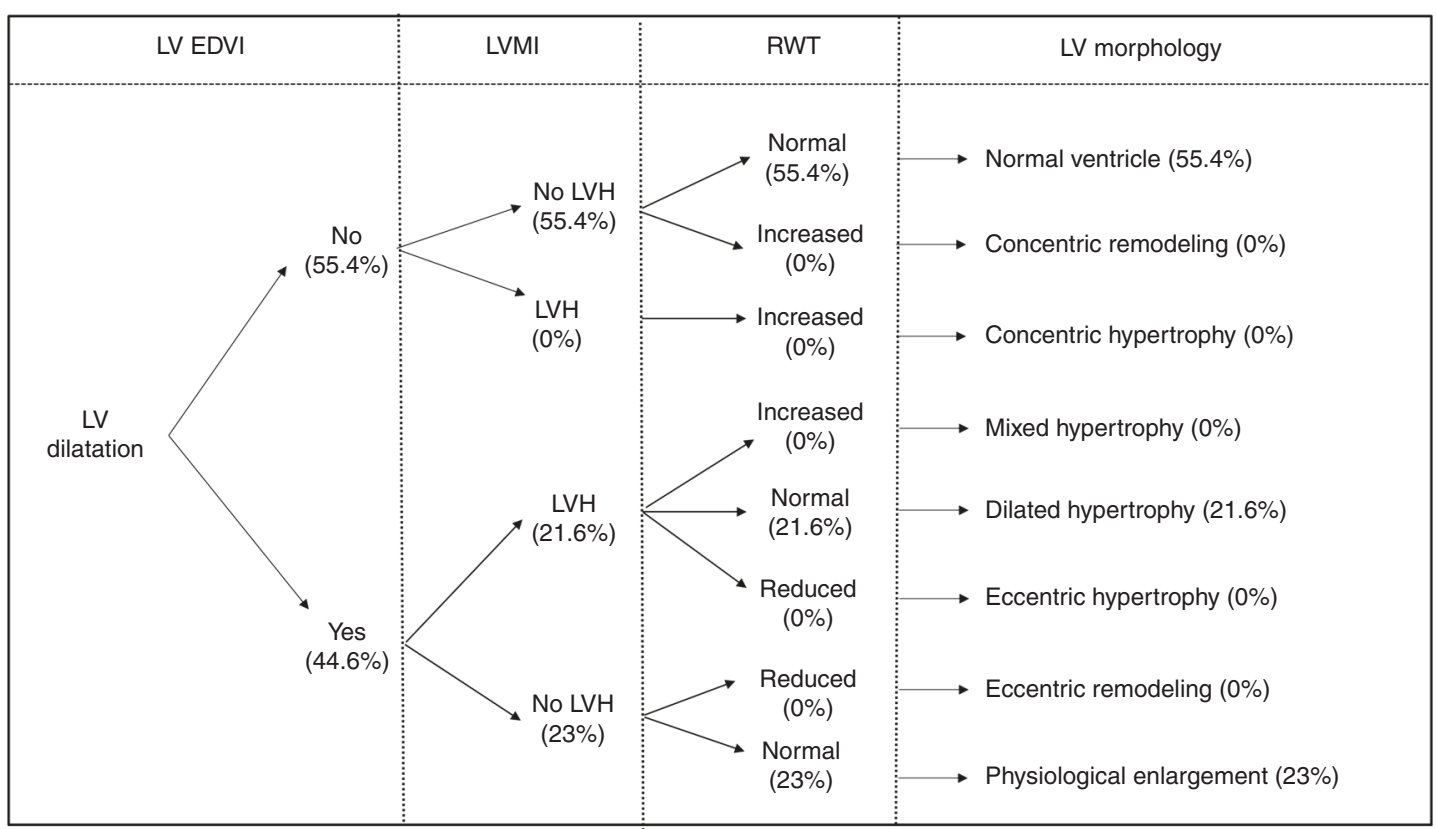

Fig. 4 Classification of the left ventricle geometry of preterm infants [adapted from Gaasch and Zile et al. ${ }^{18}$ ]. LV EDVI Left ventricle end diastolic volume index, LVMI left ventricle mass index, LVH Left ventricular hypertrophy, RWT relative wall thickness

\section{DISCUSSION}

In this study, preterm infants exposed to an average of 9 weeks of extrauterine neonatal intensive care environment showed a dilated, hypertrophic, and more spherical LV at 36 weeks PMA compared with the control infants who were exposed to the normal intrauterine environment for the same period. Although majority of the very preterm infants had normal LV geometry according to the remodeling classification, it is important to note that nearly half of them had dilatation of LV with or without hypertrophy. The data from the current study highlight that the prematurity related cardiac remodeling is evident during the neonatal intensive care period. Early detection of altered cardiac geometry and function may help identify preterm infants at increased risk for future cardiac dysfunction. ${ }^{6}$

The process of cardiac remodeling in preterm infants is inseparably linked to their preterm birth. In the fetus, multiplication of cardiomyocytes is the predominant mechanism of myocardial growth. Shortly after birth, majority cardiomyocytes become terminally differentiated and their proliferation capacity is markedly reduced. ${ }^{9,10,25}$ Consequently, hearts of infants born preterm have a smaller number of cardiomyocytes compared with infants born at term. Moreover, preterm hearts face the hemodynamic challenges of fetal to neonatal transition when their myocardium is still immature and cardiac function is not fully developed. ${ }^{13,26}$ With the expansion of lungs and onset of spontaneous breathing after birth, pulmonary blood flow increases by about 2-3 fold and about a 30\% compensatory increase occurs in the LV EDV during the transitional period in preterm infants with a PDA. ${ }^{27,28} \mathrm{~A}$ long-standing increase in LV preload leads to an increase in cardiomyocyte length due to 'in series' addition of sarcomeres and enlargement of the LV chamber with dissolution of collagen, rearrangement of cardiomyocytes and extracellular matrix. ${ }^{11-13}$ LV ventricular wall thickness increases in response to chamber enlargement in order to reduce wall stress, resulting in hypertrophy of the ventricle.

After birth, systemic vascular resistance and blood pressure also increase due to loss of the low resistance placental circulation. ${ }^{28,29}$ Pejovic et al. reported a $19 \%$ increase in the systolic blood pressure and a $34 \%$ increase in diastolic blood pressure in stable extremely preterm infants over the first week after birth. ${ }^{30}$ Consequently, comparatively higher intraventricular pressure is needed to open the aortic valve during the ejection phase, which also leads to an increase in myocardial wall stress, ventricular wall thickness, and ventricular hypertrophy. Thus, the geometric patterns of the LV in preterm infants observed in this study are consistent with the expected remodeling pattern of a ventricle facing increases in both preload and afterload.

Cardiac remodeling significantly increases the risk of cardiac failure in adults. ${ }^{17,21,31}$ In a large population based study, of all the LV geometry patterns, eccentric LV hypertrophy was associated with the highest incidence of impaired LV systolic and diastolic function. ${ }^{17}$ Zile et al. reported a two times higher prevalence of cardiac failure in older adults with dilated LV hypertrophy. ${ }^{21}$ The long term outcomes of early LV dilatation and hypertrophy associated with preterm birth are unclear at present. ${ }^{16}$ Data from well designed, prospective longitudinal studies will be valuable in understanding the association between early cardiac remodeling in preterm infants and cardiac dysfunction in the future. ${ }^{6,16}$ Several birth registry and population based preterm cohort studies are in progress, but most do not have echocardiography data around discharge from the neonatal intensive care period., ${ }^{3,6}$

Data from cross-sectional studies at various ages supports the association of cardiac remodeling and preterm birth. However, comparison of the preterm LV geometry patterns across studies to understand the progression of cardiac remodeling following preterm birth is difficult at present due to considerable differences in the reported findings. Cox et al. reported a dilated LV with increased mass in preterm infants at term equivalent age while Mohlkert et al. and Kowalski et al. reported a smaller and shorter LV with reduced LVM in children born preterm. On the other hand, Lewandowski et al. found a smaller and shorter LV with increased LVM in young adults born preterm. ${ }^{8,15,32}$ In contrast to the patterns of cardiac remodeling in adults, children born preterm did not show any difference in the RWT. ${ }^{15,32}$ Heterogeneity of the study population, use of different imaging modalities and measurements at different time points may account for the differences between the studies as cardiac remodeling is an ongoing process. Cardiac parameters essential for diagnosis and 
Dilated hypertrophy: a distinct pattern of cardiac remodeling in preterm... NS Phad et al.

\begin{tabular}{|c|c|c|c|c|c|c|}
\hline Variable/Outcome & LV dilatation & $P$-value & LV hypertrophy & $P$-value & LV dilated hypertrophy & $P$-value \\
\hline Postmenstrual age & $0.981(0.734-1.312)$ & 0.898 & $0.991(0.717-1.369)$ & 0.955 & $0.874(0.629-1.215)$ & 0.422 \\
\hline $\operatorname{Sex}(F)$ & $2.479(0.993-6.192)$ & 0.052 & $1.800(0.672-4.822)$ & 0.242 & $2.145(0.758-6.069)$ & 0.150 \\
\hline Patent ductus arteriosus $>14$ days & $0.834(0.320-2.174)$ & 0.711 & $1.571(0.555-4.447)$ & 0.394 & $1.491(0.506-4.387)$ & 0.469 \\
\hline Systolic blood pressure & $0.994(0.952-1.038)$ & 0.786 & $1.004(0.957-1.054)$ & 0.864 & $1.005(0.956-1.057)$ & 0.840 \\
\hline Diastolic blood pressure & $1.025(0.977-1.075)$ & 0.312 & $0.998(0.947-1.052)$ & 0.935 & $0.986(0.932-1.044)$ & 0.636 \\
\hline Bronchopulmonary dysplasia & $1.311(0.545-3.153)$ & 0.545 & $1.123(0.421-2.991)$ & 0.817 & $1.825(0.637-5.232)$ & 0.263 \\
\hline Postnatal steroid administration & $0.585(0.179-1.927)$ & 0.378 & $0.790(0.196-3.183)$ & 0.740 & $1.013(0.248-4.129)$ & 0.986 \\
\hline
\end{tabular}

classification of cardiac remodeling in preterm infants have not been clearly defined. Moreover, age specific echocardiography based reference values and cut off points to discriminate the parameters commonly used to assess the patterns of cardiac remodeling as normal or abnormal are not easily available in neonates. We used the mean and 2 standard deviations of parameters from the control group as partition value to classify LV geometry. Although we acknowledge that the sample was too small to be considered as reference values, our data were comparable with the findings of previous studies. Kozak-Barany et al. reported an LVMI of 37 (7) $\mathrm{g} / \mathrm{m}^{2}$ in 15 late preterm infants born between 33 and 36 weeks gestational age. ${ }^{7}$ Aye et al. reported an LV EDVI of $17(5) \mathrm{ml} / \mathrm{m}^{2}$ at 34 weeks PMA and 28 (6) $\mathrm{ml} / \mathrm{m}^{2}$ at 3 months corrected age in a cohort 93 late preterm Infants. ${ }^{5}$ The RWT reported in this study is smaller than previously reported values. ${ }^{33-35}$ The RWT was determined using M-mode in previous studies, which can explain this difference. Both M-mode and 2D methods (used in current study) are recommended by the pediatric measurements writing group of the American society of echocardiography. ${ }^{22}$

Our observation of preserved systolic function and elevated estimated LV filling pressure in the preterm infants at 36 weeks PMA is in keeping with previous reports. ${ }^{32,36-38}$ There is conflicting data about LV systolic function in preterm infants. Aye et al. reported a lower LVEF in preterm infants at baseline but not at 3 months corrected age compared with term born infants. ${ }^{5}$ On the contrary, Schubert et al. reported no difference in the LVEF and speckle tracking-derived maximal systolic myocardial velocities and LS between the preterm and control groups at term equivalent age. However, at 3 months corrected age, preterm infants had significantly reduced LV LS which probably tracks into adulthood. 8,14

None of the tested clinical covariates, such as PDA exposure and BPD had consistent influence on LV dilatation and hypertrophy at 36 weeks PMA. There was significant association between IUGR and LV hypertrophy in preterm infants. The independent influence of BW $z$-score on LVM of preterm infants has been reported during infancy but not in childhood. 5,15 There is very little data about association between infant's sex and cardiac remodeling during neonatal period. Our finding of a significant association of a complicated postnatal course with altered preterm LV geometry is broadly in keeping with the observation by Cox et al. who reported an association between the length of respiratory support and cardiac remodeling. ${ }^{16}$ Similar to this study, no significant association was seen between cardiac remodeling in preterm infants and maternal PIH, exposure to a PDA and increased blood pressure in previous reports. $8,15,16$

There are several limitations to our study. The design utilized in this study explores the impact of differences in extrauterine and intrauterine hemodynamic loads on cardiac development of preterm infants at 36 weeks PMA. It is also important to understand how the extrauterine hemodynamic load affects the geometry and function of the preterm heart with immature myocardium and physiological functional limitations compared with term heart with more mature myocardium. Due to the significant difference in the postnatal age between the two groups, the geometric patterns of preterm and term hearts following exposure to similar extrauterine hemodynamic load could not be explored in this study. Moreover, the cross-sectional design of our study provides insight into the LV geometry pattern and LV function of preterm infants at one point in time only. A longitudinal study exploring the adaptive responses of preterm and term hearts following exposure to the respective postnatal hemodynamic loads will be crucial in understanding the natural history of cardiac remodeling associated with preterm birth.

Secondly, as most preterm infants leave the tertiary neonatal intensive care unit before term corrected gestation, we analysed the LV geometry and LV function of preterm infants at 36 weeks PMA instead of full term gestation. Significant further cardiac development is expected during the last weeks of pregnancy and in early infancy. ${ }^{5,7,39}$ We have accounted for this by selecting the comparison group of a similar gestational age. Furthermore, concurrent changes in the right ventricle geometry and impact of pulmonary hypertension on LV remodeling were not explored in this study.

\section{CONCLUSION}

Hearts of preterm infants show evidence of remodeling during neonatal intensive care period. At 36 weeks, their LV is significantly dilated, hypertrophied, and more spherical compared with the PMA matched newborn infants. The association between early cardiac remodeling and higher risk of cardiovascular events at a later age in individuals born preterm needs further evaluation.

\section{ACKNOWLEDGEMENTS}

The authors thank prof John Attia for his guidance on statistical analysis. 


\section{AUTHOR CONTRIBUTION}

N.S.P. contributed to the study design, performed cardiac scans, collected and analysed data, and drafted the initial paper. K.W. contributed to study design, data collection, and revised the papers. C.H. and C.O. performed the statistical analysis and revised the paper.

\section{ADDITIONAL INFORMATION}

The online version of this article (https://doi.org/10.1038/s41390-019-0568-4) contains supplementary material, which is available to authorized users.

Financial support statement: Authors received John Hunter Hospital Charitable Trust research grant (2015) for this study. N. Phad also received the University of Newcastle research scholarship for this study.

Competing interests: The authors declare no competing interests.

Publisher's note Springer Nature remains neutral with regard to jurisdictional claims in published maps and institutional affiliations.

\section{REFERENCES}

1. Barker, D. J., Winter, P. D., Osmond, C., Margetts, B. \& Simmonds, S. J. Weight in infancy and death from ischaemic heart disease. Lancet 2, 577-80 (1989).

2. Dalziel, S. R., Parag, V., Rodgers, A. \& Harding, J. E. Cardiovascular risk factors at age 30 following pre-term birth. Int J. Epidemiol. 36, 907-15 (2007).

3. Darlow B. A., Horwood L. J., Woodward L. J. et al. The New Zealand 1986 very low birth weight cohort as young adults: mapping the road ahead. BMC Pediatr. 15 , (2015).

4. Wilson-Costello, D. Is there evidence that long-term outcomes have improved with intensive care? Semin. Fetal Neonatal Med. 12, 344-54 (2007).

5. Aye, C. Y. L., Lewandowski, A. J. \& Lamata, P. et al. Disproportionate cardiac hypertrophy during early postnatal development in infants born preterm. Pediatr. Res. 82, 36-46 (2017).

6. Carr, H., Cnattingius, S., Granath, F., Ludvigsson, J. F. \& Edstedt Bonamy, A. K. Preterm birth and risk of heart failure up to early adulthood. J. Am. Coll. Cardiol. 69, 2634-42 (2017).

7. Kozak-Barany, A., Jokinen, E., Saraste, M., Tuominen, J. \& Valimaki, I. Development of left ventricular systolic and diastolic function in preterm infants during the first month of life: a prospective follow-up study. J. Pediatr. 139, 539-45 (2001).

8. Lewandowski, A. J., Augustine, D. \& Lamata, P. et al. Preterm heart in adult life: cardiovascular magnetic resonance reveals distinct differences in left ventricular mass, geometry, and function. Circulation 127, 197-206 (2013).

9. Li, F., Wang, X., Capasso, J. M. \& Gerdes, A. M. Rapid transition of cardiac myocytes from hyperplasia to hypertrophy during postnatal development. J. Mol. Cell. Cardiol. 28, 1737-46 (1996).

10. Mayhew, T. M., Pharaoh, A., Austin, A. \& Fagan, D. G. Stereological estimates of nuclear number in human ventricular cardiomyocytes before and after birth obtained using physical disectors. J. Anat. 191(Pt 1), 107-15 (1997).

11. Bensley, J. G., Stacy, V. K., De Matteo, R., Harding, R. \& Black, M. J. Cardiac remodelling as a result of pre-term birth: implications for future cardiovascular disease. Eur. Heart J. 31, 2058-66 (2010).

12. Kehat, I. \& Molkentin, J. D. Molecular pathways underlying cardiac remodeling during pathophysiological stimulation. Circulation. 122, 2727-35 (2010).

13. Rudolph, A. M. Myocardial growth before and after birth: clinical implications. Acta Paediatr. 89, 129-33 (2000).

14. Huckstep, O. J., Williamson, W. \& Telles, F. et al. Physiological stress elicits impaired left ventricular function in preterm-born adults. J. Am. Coll. Cardiol. 71, 1347-56 (2018).

15. Mohlkert, L. A. Hallberg J., Broberg O. et al. The preterm heart in childhood: left ventricular structure, geometry, and function assessed by echocardiography in 6year-old survivors of periviable births. J Am. Heart Assoc. 7, 2018.

16. Cox D. J., Bai W., Price A. N., Edwards A. D., Rueckert D. et al. Ventricular remodeling in preterm infants: computational cardiac magnetic resonance atlasing shows significant early remodeling of the left ventricle. Pediatri. Res. 2018
17. Barbieri, A., Rossi, A. \& Gaibazzi, N. et al. Refined 4-group classification of left ventricular hypertrophy based on ventricular concentricity and volume dilatation outlines distinct noninvasive hemodynamic profiles in a large contemporary echocardiographic population. Echocardiography. 35, 1258-65 (2018).

18. Gaasch, W. H. \& Zile, M. R. Left ventricular structural remodeling in health and disease: with special emphasis on volume, mass, and geometry. J. Am. Coll. Cardiol. 58, 1733-40 (2011).

19. Khouri, M. G., Peshock, R. M., Ayers, C. R., de Lemos, J. A. \& Drazner, M. H. A 4tiered classification of left ventricular hypertrophy based on left ventricular geometry: the Dallas heart study. Circ. Cardiovasc. Imaging. 3, 164-71 (2010).

20. Marwick, T. H., Gillebert, T. C. \& Aurigemma, G. et al. Recommendations on the use of echocardiography in adult hypertension: a report from the European Association of Cardiovascular Imaging (EACVI) and the American Society of Echocardiography (ASE)dagger. Eur. Heart J. Cardiovasc. Imaging. 16, 577-605 (2015).

21. Zile, M. R., Gaasch, W. H., Patel, K., Aban, I. B. \& Ahmed, A. Adverse left ventricular remodeling in community-dwelling older adults predicts incident heart failure and mortality. JACC Heart Fail. 2, 512-22 (2014).

22. Lopez, L., Colan, S. D. \& Frommelt, P. C. et al. Recommendations for quantification methods during the performance of a pediatric echocardiogram: a report from the Pediatric Measurements Writing Group of the American Society of Echocardiography Pediatric and Congenital Heart Disease Council. J. Am. Soc. Echocardiogr. 23, 465-95 (2010).

23. de Waal, K., Phad, N., Lakkundi, A. \& Tan, P. Cardiac function after the immediate transitional period in very preterm infants using speckle tracking analysis. Pediatr. Cardiol. 37, 295-303 (2016).

24. Phad, N., de Waal, K. \& Jones, M. Agreement and reliability of the velocity time integral method and the method of disks to determine stroke volume in preterm infants. Early Hum. Dev. 125, 31-4 (2018).

25. Bergmann, O., Zdunek, S. \& Felker, A. et al. Dynamics of cell generation and turnover in the human heart. Cell 161, 1566-75 (2015).

26. Friedman, W. F. The intrinsic physiologic properties of the developing heart. Prog. Cardiovasc. Dis. 15, 87-111 (1972).

27. de Waal, K., Phad, N., Collins, N. \& Boyle, A. Cardiac remodeling in preterm infants with prolonged exposure to a patent ductus arteriosus. Congenit. Heart Dis. 12, 364-72 (2017).

28. Hooper, S. B., Te Pas, A. B., Lang, J. \& van Vonderen, J. J. et al. Cardiovascular transition at birth: a physiological sequence. Pediatr. Res. 77, 608-14 (2015).

29. Morton, S. U. \& Brodsky, D. Fetal physiology and the transition to extrauterine life. Clin. Perinatol. 43, 395-407 (2016).

30. Pejovic, B., Peco-Antic, A. \& Marinkovic-Eric, J. Blood pressure in non-critically ill preterm and full-term neonates. Pediatr. Nephrol. 22, 249-57 (2007).

31. Krumholz, H. M., Larson, M. \& Levy, D. Prognosis of left ventricular geometric patterns in the Framingham Heart Study. J. Am. Coll. Cardiol. 25, 879-84 (1995).

32. Kowalski, R. R., Beare, R., Doyle, L. W., Smolich, J. J. \& Cheung, M. M. Victorian infant collaborative study. elevated blood pressure with reduced left ventricular and aortic dimensions in adolescents born extremely preterm. J. Pediatr. 172, e2 (2016).

33. Choudhry, S., Salter, A. \& Cunningham, T. W. et al. Normative left ventricular MMode echocardiographic values in preterm infants up to $2 \mathrm{~kg}$. J. Am. Soc. Echocardiogr. 30, 781-9 e4 (2017).

34. Guzeltas, A. \& Eroglu, A. G. Reference values for echocardiographic measurements of healthy newborns. Cardiol. Young. 22, 152-7 (2012).

35. Kampmann, C., Wiethoff, C. M. \& Wenzel, A. et al. Normal values of M mode echocardiographic measurements of more than 2000 healthy infants and children in central Europe. Heart 83, 667-72 (2000).

36. Di Maria, M. V., Younoszai, A. K. \& Sontag, M. K. et al. Maturational changes in diastolic longitudinal myocardial velocity in preterm infants. J. Am. Soc. Echocardiogr. 28, 1045-52 (2015).

37. Hirose, A., Khoo, N. S. \& Aziz, K. et al. Evolution of left ventricular function in the preterm infant. J. Am. Soc. Echocardiogr. 28, 302-8 (2015).

38. Schubert, U., Muller, M., Abdul-Khaliq, H. \& Norman, M. Preterm birth is associated with altered myocardial function in infancy. J. Am. Soc. Echocardiogr. 29, 670-8 (2016).

39. Joyce, J. J., Dickson, P. I., Qi, N., Noble, J. E., Raj, J. U. \& Baylen, B. G. Normal right and left ventricular mass development during early infancy. Am. J. Cardiol. 93, 797-801 (2004). 